\title{
Shape invariance, raising and lowering operators in hypergeometric type equations
}

\author{
Nicolae Cotfas \\ Faculty of Physics, University of Bucharest, PO Box 76-54, Postal Office 76, \\ Bucharest, Romania, E-mail address: ncotfas@yahoo.com

\begin{abstract}
The Schrödinger equations which are exactly solvable in terms of associated special functions are directly related to some self-adjoint operators defined in the theory of hypergeometric type equations. The fundamental formulae occurring in a supersymmetric approach to these Hamiltonians are consequences of some formulae concerning the general theory of associated special functions. We use this connection in order to obtain a general theory of Schrödinger equations exactly solvable in terms of associated special functions, and to extend certain results known in the case of some particular potentials.
\end{abstract}

Short title: Shape invariance, raising and lowering operators

October 25, 2018 


\section{Introduction}

It is well-known 10, 50 that, in the case of certain potentials, the Schrödinger equation is exactly solvable and its solutions can be expressed in terms of the socalled associated special functions (ASF). The Hamiltonian of such a system can be factorized as a product of two first order differential operators, and a hierarchy of almost isospectral Hamiltonians (called supersymmetric partners) can be defined by repeated refactorizations.

The hierarchy of Hamiltonians corresponds to a hierarchy of self-adjoint operators having the corresponding ASF as eigenfunctions, and the first order differential operators involved in their factorizations correspond to some first order differential operators relating ASF. The purpose of the present article is to analyse in a unitary way the quantum systems exactly solvable in terms of ASF. In order to do this, we start from the general theory of hypergeometric type equations [18], and factorize the self-adjoint operators having the ASF as eigenfunctions by using some raising/lowering operators relating these functions.

\section{Orthogonal polynomials and ASF}

Many problems in quantum mechanics and mathematical physics lead to equations of hypergeometric type

$$
\sigma(s) y^{\prime \prime}(s)+\tau(s) y^{\prime}(s)+\lambda y(s)=0
$$

where $\sigma(s)$ and $\tau(s)$ are polynomials of at most second and first degree, respectively, and $\lambda$ is a constant. These equations are usually called equations of hypergeometric type, and the corresponding solutions functions of hypergeometric type [18]. The equation (11) can be reduced to the self-adjoint form

$$
\left[\sigma(s) \varrho(s) y^{\prime}(s)\right]^{\prime}+\lambda \varrho(s) y(s)=0
$$

by choosing a function $\varrho$ such that $[\sigma(s) \varrho(s)]^{\prime}=\tau(s) \varrho(s)$. For

$$
\lambda=\lambda_{l}=-\frac{1}{2} l(l-1) \sigma^{\prime \prime}-l \tau^{\prime} \quad \text { with } l \in \mathbb{N}
$$

there exists a polynomial $\Phi_{l}$ of degree $l$ satisfying (1), that is,

$$
\sigma(s) \Phi_{l}^{\prime \prime}(s)+\tau(s) \Phi_{l}^{\prime}(s)+\lambda_{l} \Phi_{l}(s)=0 .
$$

If there exists a finite or infinite interval $(a, b)$ such that

$$
\left.\sigma(s) \varrho(s) s^{k}\right|_{s=a}=\left.0 \quad \sigma(s) \varrho(s) s^{k}\right|_{s=b}=0 \quad \text { for all } k \in \mathbb{N}
$$

and if $\sigma(s)>0, \varrho(s)>0$ for all $s \in(a, b)$, then the polynomials $\Phi_{l}$ are orthogonal with weight function $\varrho(s)$ in the interval $(a, b)$

$$
\int_{a}^{b} \Phi_{l}(s) \Phi_{k}(s) \varrho(s) d s=0 \quad \text { for } \lambda_{l} \neq \lambda_{k} .
$$

In this case $\Phi_{l}$ are known as classical orthogonal polynomials 18 . We shall prove that the condition $\lambda_{l} \neq \lambda_{k}$ from (6) can be replaced by $l \neq k$. The main particular cases of this general approach are presented in table 1. 


\begin{tabular}{||c|c|c|c|c||}
\hline \hline Name & $(a, b)$ & $\sigma(s)$ & $\tau(s)$ & $\varrho(s)$ \\
\hline \hline Hypergeometric & $(0,1)$ & $s(1-s)$ & $(\alpha+1)-(\alpha+\beta+2) s$ & $s^{\alpha}(1-s)^{\beta}$ \\
\hline Jacobi & $(-1,1)$ & $1-s^{2}$ & $(\beta-\alpha)-(\alpha+\beta+2) s$ & $(1-s)^{\alpha}(1+s)^{\beta}$ \\
\hline Laguerre & $(0, \infty)$ & $s$ & $\alpha+1-s$ & $s^{\alpha} \mathrm{e}^{-s}$ \\
\hline Hermite & $(-\infty, \infty)$ & 1 & $-s$ & $\mathrm{e}^{-s^{2}}$ \\
\hline \hline
\end{tabular}

Table 1. Some important particular cases (the parameters $\alpha, \beta$ belong to $(-1, \infty)$ ).

The classical orthogonal polynomials $\Phi_{l}$ satisfy a three term recurrence relation

$$
s \Phi_{l}(s)=\alpha_{l} \Phi_{l+1}(s)+\beta_{l} \Phi_{l}(s)+\gamma_{l} \Phi_{l-1}(s) .
$$

and Rodrigues formula

$$
\Phi_{l}(s)=\frac{B_{l}}{\varrho(s)}\left[\sigma^{l}(s) \varrho(s)\right]^{(l)}
$$

where $\alpha_{l}, \beta_{l}, \gamma_{l}$ and $B_{l}$ are constants [18].

Let $\kappa(s)=\sqrt{\sigma(s)}$. By differentiating the equation (4) $m$ times and multiplying it by $\kappa^{m}(s)$, we get for each $m \in\{0,1,2, \ldots, l\}$ the associated differential equation

$$
\begin{aligned}
-\sigma(s) \Phi_{l, m}^{\prime \prime}-\tau(s) \Phi_{l, m}^{\prime} & +\left[\frac{m(m-2)}{4} \frac{\sigma^{\prime 2}(s)}{\sigma(s)}+\frac{m \tau(s)}{2} \frac{\sigma^{\prime}(s)}{\sigma(s)}\right. \\
& \left.-\frac{1}{2} m(m-2) \sigma^{\prime \prime}(s)-m \tau^{\prime}(s)\right] \Phi_{l, m}=\lambda_{l} \Phi_{l, m}
\end{aligned}
$$

where

$$
\Phi_{l, m}(s)=\kappa^{m}(s) \Phi_{l}^{(m)}(s)
$$

are known as the associated special functions. We have ([18], p.8)

$$
\int_{a}^{b} \Phi_{l, m}(s) \Phi_{k, m}(s) \varrho(s) d s=\int_{a}^{b} \Phi_{l}^{(m)}(s) \Phi_{k}^{(m)}(s) \sigma^{m}(s) \varrho(s) d s=0
$$

for any $m \in \mathbb{N}$ and $l, k \in\{m, m+1, m+2, \ldots\}$ with $l \neq k$. This means that for each $m \in \mathbb{N}$, the set $\left\{\Phi_{m, m}, \Phi_{m+1, m}, \Phi_{m+2, m}, \ldots\right\}$ (se figure 1) is an orthogonal sequence in the Hilbert space

$$
\mathcal{H}=\left\{\varphi:\left.(a, b) \longrightarrow \mathbb{R}\left|\int_{a}^{b}\right| \varphi(s)\right|^{2} \varrho(s) d s<\infty\right\}
$$

with the scalar product given by

$$
\langle\varphi, \psi\rangle=\int_{a}^{b} \varphi(s) \psi(s) \varrho(s) d s .
$$

For each $m \in \mathbb{N}$, let $\mathcal{H}_{m}$ be the linear span of $\left\{\Phi_{m, m}, \Phi_{m+1, m}, \Phi_{m+2, m}, \ldots\right\}$. In the sequel we shall restrict us to the case when $\mathcal{H}_{m}$ is dense in $\mathcal{H}$ for all $m \in \mathbb{N}$. For this it is sufficient the interval $(a, b)$ to be finite, but not necessary.

The equation (9) can be written as

$$
H_{m} \Phi_{l, m}=\lambda_{l} \Phi_{l, m}
$$




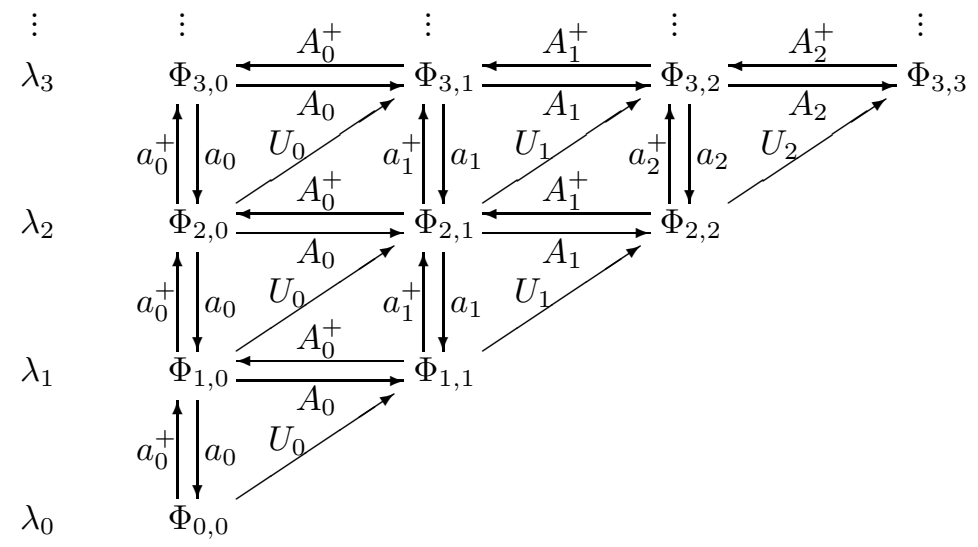

Figure 1. The functions $\Phi_{l, m}$ satisfy the relation $H_{m} \Phi_{l, m}=\lambda_{l} \Phi_{l, m}$, and are related (up to some multiplicative constants) through the operators $A_{m}, A_{m}^{+}, a_{m}, a_{m}^{+}, U_{m}$ and $U_{m}^{-1}=U_{m}^{+}$.

where $H_{m}: \mathcal{H}_{m} \longrightarrow \mathcal{H}_{m}$ is the differential operator

$$
\begin{aligned}
H_{m}= & -\sigma(s) \frac{d^{2}}{d s^{2}}-\tau(s) \frac{d}{d s}+\frac{m(m-2)}{4} \frac{\sigma^{\prime 2}(s)}{\sigma(s)} \\
& +\frac{m \tau(s)}{2} \frac{\sigma^{\prime}(s)}{\sigma(s)}-\frac{1}{2} m(m-2) \sigma^{\prime \prime}(s)-m \tau^{\prime}(s) .
\end{aligned}
$$

The problem of factorization of operators $H_{m}$ is a very important one since it is directly related to the factorization of some Schrödinger type operators [10, 5]. If we use in (9) a change of variable $s=s(x)$ such that $d s / d x=\kappa(s(x))$ or $d s / d x=-\kappa(s(x))$ and define the new functions

$$
\Psi_{l, m}(x)=\sqrt{\kappa(s(x)) \varrho(s(x))} \Phi_{l, m}(s(x))
$$

then we get an equation of Schrödinger type

$$
-\frac{d^{2}}{d x^{2}} \Psi_{l, m}(x)+V_{m}(x) \Psi_{l, m}(x)=\lambda_{l} \Psi_{l, m}(x) .
$$

For example, by starting from the equation of Jacobi polynomials with $\alpha=\mu-1 / 2$, $\beta=\eta-1 / 2$, and using the change of variable $s(x)=\cos x$ we obtain the Schrödinger equation corresponding to the Pöschl-Teller potential

$$
V_{0}(x)=\frac{1}{4}\left[\frac{\mu(\mu-1)}{\cos ^{2}(x / 2)}+\frac{\eta(\eta-1)}{\sin ^{2}(x / 2)}\right]-\frac{(\mu+\eta)^{2}}{4} .
$$

\section{Raising and lowering operators. Shape invariance}

Lorente has shown recently [15, 16] that a factorization of $H_{0}$ can be obtained by using the three term recurrence relation (8) and a consequence of Rodrigues formula. Following Lorente's idea we obtain a factorization of $H_{m}$ by using (10) and a three term recurrence relation. 
Theorem 1. For any $l \in \mathbb{N}$ and any $m \in\{0,1, \ldots, l-1\}$ we have

$$
\Phi_{l, m+1}(s)=\left(\kappa(s) \frac{d}{d s}-m \kappa^{\prime}(s)\right) \Phi_{l, m}(s) .
$$

Proof. By differentiating (10) we get

$$
\Phi_{l, m}^{\prime}(s)=m \kappa^{m-1}(s) \kappa^{\prime}(s) \Phi_{l}^{(m)}+\kappa^{m}(s) \Phi_{l}^{(m+1)}(s)
$$

that is, the relation

$$
\Phi_{l, m}^{\prime}(s)=m \frac{\kappa^{\prime}(s)}{\kappa(s)} \Phi_{l, m}(s)+\frac{1}{\kappa(s)} \Phi_{l, m+1}(s)
$$

equivalent to $(19)$.

Theorem 2. The three term recurrence relation

$\Phi_{l, m+1}(s)+\left(\frac{\tau(s)}{\kappa(s)}+2(m-1) \kappa^{\prime}(s)\right) \Phi_{l, m}(s)+\left(\lambda_{l}-\lambda_{m-1}\right) \Phi_{l, m-1}(s)=0$

is satisfied for any $l \in \mathbb{N}$ and any $m \in\{1,2, \ldots, l-1\}$.

Proof. In order to obtain (20), one has to differentiate (4) $m-1$ times, to multiply the obtained relation by $\kappa^{m-1}(s)$, and then to use (10).

Theorem 3. For any $l \in \mathbb{N}$ and any $m \in\{1,2, \ldots, l-1\}$ we have the relation

$$
\left(\lambda_{l}-\lambda_{m}\right) \Phi_{l, m}(s)=\left(-\kappa(s) \frac{d}{d s}-\frac{\tau(s)}{\kappa(s)}-(m-1) \kappa^{\prime}(s)\right) \Phi_{l, m+1}(s) .
$$

Proof. This relation follows from (20) and (19) with $m+1$ instead of $m$.

Theorem 4. The operators

$$
A_{m}: \mathcal{H}_{m} \longrightarrow \mathcal{H}_{m+1} \quad A_{m}=\kappa(s) \frac{d}{d s}-m \kappa^{\prime}(s)
$$

and

$A_{m}^{+}: \mathcal{H}_{m+1} \longrightarrow \mathcal{H}_{m} \quad A_{m}^{+}=-\kappa(s) \frac{d}{d s}-\frac{\tau(s)}{\kappa(s)}-(m-1) \kappa^{\prime}(s)$

are mutually adjoint [19].

Proof. Since $\sigma^{m}(s) \Phi_{l}^{(m)}(s) \Phi_{k}^{(m+1)}(s)$ is a polynomial, from (5) we get

$$
\begin{aligned}
& \left\langle A_{m} \Phi_{l, m}, \Phi_{k, m+1}\right\rangle=\int_{a}^{b}\left[\kappa(s) \Phi_{l, m}^{\prime}(s)-m \kappa^{\prime}(s) \Phi_{l, m}(s)\right] \Phi_{k, m+1}(s) \varrho(s) d s \\
& =\left.\kappa(s) \Phi_{l, m}(s) \Phi_{k, m+1}(s) \varrho(s)\right|_{a} ^{b}-\int_{a}^{b} \Phi_{l, m}(s)\left[\kappa(s) \Phi_{k, m+1}^{\prime}(s) \varrho(s)\right. \\
& \left.+\kappa(s) \Phi_{k, m+1}(s) \varrho^{\prime}(s)+(m+1) \kappa^{\prime}(s) \Phi_{k, m+1}(s) \varrho(s)\right] d s \\
& =\left.\sigma(s) \varrho(s) \sigma^{m}(s) \Phi_{l}^{(m)}(s) \Phi_{k}^{(m+1)}(s)\right|_{a} ^{b}+\int_{a}^{b} \Phi_{l, m}(s)\left(A_{m}^{+} \Phi_{k, m+1}\right)(s) \varrho(s) d s
\end{aligned}
$$




$$
=\left\langle\Phi_{l, m}, A_{m}^{+} \Phi_{k, m+1}\right\rangle
$$

for any $l \geq m, k \geq m+1$.

Since

$$
\begin{aligned}
\left\|\Phi_{l, m+1}\right\|^{2} & =\left\langle\Phi_{l, m+1}, \Phi_{l, m+1}\right\rangle=\left\langle A_{m} \Phi_{l, m}, \Phi_{l, m+1}\right\rangle \\
& =\left\langle\Phi_{l, m}, A_{m}^{+} \Phi_{l, m+1}\right\rangle=\left(\lambda_{l}-\lambda_{m}\right)\left\|\Phi_{l, m}\right\|^{2}
\end{aligned}
$$

it follows that $\lambda_{l}>\lambda_{m}$ for all $l>m$, and

$$
\left\|\Phi_{l, m+1}\right\|=\sqrt{\lambda_{l}-\lambda_{m}}\left\|\Phi_{l, m}\right\| \text {. }
$$

This is possible only if $\sigma^{\prime \prime}(s) \leq 0$ and $\tau^{\prime}(s)<0$. Particularly, we have $\lambda_{l} \neq \lambda_{k}$ if and only if $l \neq k$.

Theorem 5. The operators $H_{m}: \mathcal{H}_{m} \longrightarrow \mathcal{H}_{m}$ are self-adjoint, and

$$
H_{m}-\lambda_{m}=A_{m}^{+} A_{m} \quad H_{m+1}-\lambda_{m}=A_{m} A_{m}^{+} .
$$

Proof. The relations (19) and (21) can be written as

$$
A_{m} \Phi_{l, m}=\Phi_{l, m+1} \quad A_{m}^{+} \Phi_{l, m+1}=\left(\lambda_{l}-\lambda_{m}\right) \Phi_{l, m}
$$

and we get

$A_{m}^{+} A_{m} \Phi_{l, m}=\left(\lambda_{l}-\lambda_{m}\right) \Phi_{l, m} \quad A_{m} A_{m}^{+} \Phi_{l, m+1}=\left(\lambda_{l}-\lambda_{m}\right) \Phi_{l, m+1}$

that is,

$$
\left(A_{m}^{+} A_{m}+\lambda_{m}\right) \Phi_{l, m}=\lambda_{l} \Phi_{l, m} \quad\left(A_{m} A_{m}^{+}+\lambda_{m}\right) \Phi_{l, m+1}=\lambda_{l} \Phi_{l, m+1}
$$

whence

$$
H_{m}=A_{m}^{+} A_{m}+\lambda_{m} \quad H_{m+1}=A_{m} A_{m}^{+}+\lambda_{m} .
$$

From (25) we obtain the relation expressing the shape invariance [2, 8, 7] of operators $H_{m}$

$$
A_{m} A_{m}^{+}=A_{m+1}^{+} A_{m+1}+r_{m+1}
$$

where $r_{m+1}=\lambda_{m+1}-\lambda_{m}=-m \sigma^{\prime \prime}-\tau^{\prime}$. Particularly, we have $\lambda_{l}=\sum_{k=1}^{l} r_{k}$ and

$$
\begin{aligned}
& H_{0}=A_{0}^{+} A_{0} \\
& H_{1}=A_{0} A_{0}^{+}=A_{1}^{+} A_{1}+r_{1} \\
& H_{2}=A_{1} A_{1}^{+}+r_{1}=A_{2}^{+} A_{2}+r_{1}+r_{2} \\
& \ldots \\
& H_{m+1}=A_{m} A_{m}^{+}+\sum_{k=1}^{m} r_{k}=A_{m+1}^{+} A_{m+1}+\sum_{k=1}^{m+1} r_{k} \\
& \ldots .
\end{aligned}
$$

The function $\Phi_{l, l}(s)=\kappa^{l}(s) \Phi_{l}^{(l)}(s)$ satisfies the relation $A_{l} \Phi_{l, l}=0$, and

$$
\Phi_{l, m}=\frac{A_{m}^{+}}{\lambda_{l}-\lambda_{m}} \frac{A_{m+1}^{+}}{\lambda_{l}-\lambda_{m+1}} \ldots \frac{A_{l-2}^{+}}{\lambda_{l}-\lambda_{l-2}} \frac{A_{l-1}^{+}}{\lambda_{l}-\lambda_{l-1}} \Phi_{l, l}
$$

for all $l \in \mathbb{N}$ and $m \in\{0,1,2, \ldots, l-1\}$. 
The operators $A_{m}$ and $A_{m}^{+}$have been previously obtained by Jafarizadeh and Fakhri [1] after a rather long calculation by using the ansatz

$$
A_{m}=f_{1}(s) \frac{d}{d s}+g_{1}(s) \quad A_{m}^{+}=f_{2}(s) \frac{d}{d s}+g_{2}(s) .
$$

We use this opportunity to correct a minor error existing in [11]. Since Jafarizadeh and Fakhri [11] use for ASF the definition $\Phi_{l, m}(s)=(-1)^{m} \kappa^{m}(s) \Phi_{l}^{(m)}(s)$, the proof of our theorem 1 shows that one has to multiply the expressions of $B_{-}(m)$ and $A_{-}(m)$ from [11 by $(-1)$ in order to get the correct raising/lowering operators. The expression of $A_{m}$ in the Legendre case is known for a long time [20].

\section{Creation and annihilation operators}

For each $m \in \mathbb{N}$, the sequence $\{|m, m>| m+1,, m>, \mid m+2, m>, \ldots\}$, where

$$
\mid l, m>=\Phi_{l, m} /\left\|\Phi_{l, m}\right\|
$$

is an orthonormal basis of $\mathcal{H}$, and

$$
U_{m}: \mathcal{H} \longrightarrow \mathcal{H} \quad U_{m}|l, m\rangle=|l+1, m+1\rangle
$$

is a unitary operator.

Theorem 6. The operators (see figure 1)

$a_{m}, a_{m}^{+}: \mathcal{H}_{m} \longrightarrow \mathcal{H}_{m} \quad a_{m}=U_{m}^{+} A_{m} \quad a_{m}^{+}=A_{m}^{+} U_{m}$

are mutually adjoint, and

$$
\begin{array}{ll}
a_{m}|l, m\rangle=\sqrt{\lambda_{l}-\lambda_{m}}|l-1, m\rangle \quad \text { for all } & l \geq m+1 \\
a_{m}^{+}|l, m\rangle=\sqrt{\lambda_{l+1}-\lambda_{m}}|l+1, m\rangle \quad \text { for all } \quad l \geq m .
\end{array}
$$

Proof. This result follows from (24) and the fact that $A_{m}$ and $A_{m}^{+}$are mutually adjoint.

For each $l>m$ we have

$$
|l, m\rangle=\frac{\left(a_{m}^{+}\right)^{l-m}}{\sqrt{\left(\lambda_{l}-\lambda_{m}\right)\left(\lambda_{l-1}-\lambda_{m}\right) \ldots\left(\lambda_{m+1}-\lambda_{m}\right)}}|m, m\rangle .
$$

Since

$a_{m} a_{m}^{+} \Phi_{l, m}=\left(\lambda_{l+1}-\lambda_{m}\right) \Phi_{l, m} \quad a_{m}^{+} a_{m} \Phi_{l+1, m}=\left(\lambda_{l+1}-\lambda_{m}\right) \Phi_{l+1, m}$

we get the factorization

$$
H_{m}-\lambda_{m}=a_{m}^{+} a_{m}
$$

and the relation

$$
\left[a_{m}, a_{m}^{+}\right] \Phi_{l, m}=\left(\lambda_{l+1}-\lambda_{l}\right) \Phi_{l, m} .
$$

By using the operator $R_{m}=-\sigma^{\prime \prime} N_{m}-\tau^{\prime}$, where $N_{m}$ is the number operator

$$
N_{m}: \mathcal{H}_{m} \longrightarrow \mathcal{H}_{m} \quad N_{m} \Phi_{l, m}=l \Phi_{l, m}
$$


the relation (39) can be written as 6, 2]

$$
\left[a_{m}, a_{m}^{+}\right]=R_{m} .
$$

Since

$$
\left[a_{m}^{+}, R_{m}\right]=\sigma^{\prime \prime} a_{m}^{+} \quad\left[a_{m}, R_{m}\right]=-\sigma^{\prime \prime} a_{m}
$$

it follows that the Lie algebra $\mathcal{L}_{m}$ generated by $\left\{a_{m}^{+}, a_{m}\right\}$ is finite dimensional.

\section{Theorem 7.}

$\mathcal{L}_{m}$ is isomorphic to $\begin{cases}\text { su }(1,1) & \text { if } \quad \sigma^{\prime \prime}<0 \\ \text { Heisenberg -Weyl algebra } & \text { if } \quad \sigma^{\prime \prime}=0 .\end{cases}$

Proof. If $\sigma^{\prime \prime} \neq 0$ then $K_{+}=\sqrt{2 /\left|\sigma^{\prime \prime}\right|} a_{m}^{+}, K_{-}=\sqrt{2 /\left|\sigma^{\prime \prime}\right|} a_{m}$ and $K_{0}=\left(-1 / \sigma^{\prime \prime}\right) R_{m}$ satisfy

$$
\left[K_{0}, K_{ \pm}\right]= \pm K_{ \pm} \quad\left[K_{+}, K_{-}\right]=-2 K_{0} .
$$

By using (34) the relation (41) can be written as

$$
U_{m}^{+} A_{m} A_{m}^{+} U_{m}-A_{m}^{+} U_{m} U_{m}^{+} A_{m}=R_{m} .
$$

and in view of (59) we get

$$
U_{m}^{+}\left(H_{m+1}-\lambda_{m}\right) U_{m}-\left(H_{m}-\lambda_{m}\right)=R_{m}
$$

that is, the relation expressing the shape invariance [8] of $H_{m}$

$$
H_{m+1}=U_{m}\left(H_{m}+R_{m}\right) U_{m}^{+} .
$$

One can also remark that

$$
\begin{array}{ll}
A_{m} R_{m}=R_{m+1} A_{m} & R_{m} A_{m}^{+}=A_{m}^{+} R_{m+1} \\
{\left[H_{m}, a_{m}\right]=-R_{m} a_{m}} & {\left[H_{m}, a_{m}^{+}\right]=a_{m}^{+} R_{m}}
\end{array}
$$

and

$$
U_{m} R_{m} U_{m}^{+}=R_{m+1}+\sigma^{\prime \prime}
$$

for all $m \in \mathbb{N}$.

\section{Systems of coherent states}

Let $m \in \mathbb{N}$ be a fixed natural number, and let

$$
|n\rangle=|m+n, m\rangle \quad e_{n}=\lambda_{m+n}-\lambda_{m}
$$

for all $n \in \mathbb{N}$. Since

$$
0=e_{0}<e_{1}<e_{2}<\ldots<e_{n}<\ldots
$$

and

$a_{m}|n\rangle=\sqrt{e_{n}}|n-1\rangle \quad a_{m}^{+}|n\rangle=\sqrt{e_{n+1}}|n+1\rangle \quad\left(H_{m}-\lambda_{m}\right)|n\rangle=e_{n}|n\rangle$ 
we can define a system of coherent states by using the general setting presented in [1].

Let

$$
\varepsilon_{n}=\left\{\begin{array}{lll}
1 & \text { if } & n=0 \\
e_{1} e_{2} \ldots e_{n} & \text { if } & n>0
\end{array}\right.
$$

If

$$
R=\limsup _{n \rightarrow \infty} \sqrt[n]{\varepsilon_{n}} \neq 0
$$

then we can define

$|z\rangle=\frac{1}{N\left(|z|^{2}\right)} \sum_{n \geq 0} \frac{z^{n}}{\sqrt{\varepsilon_{n}}}|n\rangle \quad$ where $\quad\left(N\left(|z|^{2}\right)^{2}=\sum_{n=0}^{\infty} \frac{|z|^{2 n}}{\varepsilon_{n}}\right.$

for any $z$ in the open disk $C(0, R)$ of center 0 and radius $R$. We get in this way a continuous family $\{|z\rangle \mid z \in C(0, R)\}$ of normalized coherent states, eigenstates of the operator $a_{m}$

$$
a_{m}|z\rangle=z|z\rangle .
$$

\section{Application to Schrödinger type operators}

We have already seen that the operators $H_{m}$ are directly related to some Schrödinger type operators. If we use a change of variable $s=s(x)$ such that $d s / d x=\kappa(s(x))$, then the operators corresponding to $A_{m}$ and $A_{m}^{+}$are the adjoint conjugate operators

$$
\begin{aligned}
& \tilde{A}_{m}=\left.[\kappa(s) \varrho(s)]^{1 / 2} A_{m}[\kappa(s) \varrho(s)]^{-1 / 2}\right|_{s=s(x)}=\frac{d}{d x}+W_{m}(x) \\
& \tilde{A}_{m}^{+}=\left.[\kappa(s) \varrho(s)]^{1 / 2} A_{m}^{+}[\kappa(s) \varrho(s)]^{-1 / 2}\right|_{s=s(x)}=-\frac{d}{d x}+W_{m}(x)
\end{aligned}
$$

where the superpotential $W_{m}(x)$ is given by the formula

$$
W_{m}(x)=-\frac{\tau(s(x))}{2 \kappa(s(x))}-\frac{2 m-1}{2 \kappa(s(x))} \frac{d}{d x} \kappa(s(x)) .
$$

From (26) and (27) we get

$$
\tilde{A}_{m} \Psi_{l, m}(x)=\Psi_{l, m+1}(x) \quad \tilde{A}_{m}^{+} \Psi_{l, m+1}(x)=\left(\lambda_{l}-\lambda_{m}\right) \Psi_{l, m}(x)
$$

and

$\left(\tilde{A}_{m}^{+} \tilde{A}_{m}+\lambda_{m}\right) \Psi_{l, m}=\lambda_{l} \Psi_{l, m} \quad\left(\tilde{A}_{m} \tilde{A}_{m}^{+}+\lambda_{m}\right) \Psi_{l, m+1}=\lambda_{l} \Psi_{l, m+1}$

whence

$$
-\frac{d^{2}}{d x^{2}}+V_{m}(x)-\lambda_{m}=\tilde{A}_{m}^{+} \tilde{A}_{m} \quad-\frac{d^{2}}{d x^{2}}+V_{m+1}(x)-\lambda_{m}=\tilde{A}_{m} \tilde{A}_{m}^{+}
$$

and

$V_{m}(x)-\lambda_{m}=W_{m}^{2}(x)-\dot{W}_{m}(x) \quad V_{m+1}(x)-\lambda_{m}=W_{m}^{2}(x)+\dot{W}_{m}(x)$

where the dot sign means derivative with respect to $x$.

Since $\tilde{A}_{m} \Psi_{m, m}=0$, from (55) and (59) we get

$\dot{\Psi}_{m, m}+W_{m}(x) \Psi_{m, m}=0 \quad-\ddot{\Psi}_{m, m}+\left(V_{m}(x)-\lambda_{m}\right) \Psi_{m, m}=0$ 
whence

$$
W_{m}(x)=-\frac{\dot{\Psi}_{m, m}(x)}{\Psi_{m, m}(x)} \quad V_{m}(x)=\frac{\ddot{\Psi}_{m, m}(x)}{\Psi_{m, m}(x)}+\lambda_{m} .
$$

For each $m \in\{0,1,2, \ldots, l-1\}$ we have

$$
\Psi_{l, m}(x)=\frac{\tilde{A}_{m}^{+}}{\lambda_{l}-\lambda_{m}} \frac{\tilde{A}_{m+1}^{+}}{\lambda_{l}-\lambda_{m+1}} \ldots \frac{\tilde{A}_{l-2}^{+}}{\lambda_{l}-\lambda_{l-2}} \frac{\tilde{A}_{l-1}^{+}}{\lambda_{l}-\lambda_{l-1}} \Psi_{l, l}(x) .
$$

If we choose the change of variable $s=s(x)$ such that $d s / d x=-\kappa(s(x))$, then the formulae (55), (56), (60) and (62) become

$$
\begin{gathered}
\tilde{A}_{m}=-\frac{d}{d x}+W_{m}(x) \quad \tilde{A}_{m}^{+}=\frac{d}{d x}+W_{m}(x) \\
W_{m}(x)=-\frac{\tau(s(x))}{2 \kappa(s(x))}+\frac{2 m-1}{2 \kappa(s(x))} \frac{d}{d x} \kappa(s(x)) \\
V_{m}(x)-\lambda_{m}=W_{m}^{2}(x)+\dot{W}_{m}(x) \quad V_{m+1}(x)-\lambda_{m}=W_{m}^{2}(x)-\dot{W}_{m}(x) \\
W_{m}(x)=\frac{\dot{\Psi}_{m, m}(x)}{\Psi_{m, m}(x)}
\end{gathered}
$$

respectively. For example, in the case of Pöschl-Teller potential (18) we obtain

$$
W_{0}(x)=\frac{1}{2}\left[\mu \cot \frac{x}{2}-\eta \tan \frac{x}{2}\right] .
$$

\section{Concluding remarks}

The Schrödinger equations which are exactly solvable in terms of ASF are directly related to the self-adjoint operators $H_{m}$ defined by (15), and hence, each of them can be described by the interval $(a, b)$ and the corresponding functions $\sigma(s), \tau(s), \varrho(s), s(x)$ satisfying the conditions presented in section 2 . This infinite class of exactly solvable problems depending on several parameters contains well-known potentials (together with their supersymmetric partners) as well as other physically relevant potentials [11.

Our results concerning the operators $H_{m}$ allow to study these quantum systems together in a unitary way, and to extend certain results presented up to now only in case of some particular potentials. Our formulae apply to all the Schrödinger equations solvable in terms of ASF. In order to pass to a particular potential it suffices to replace $\sigma(s), \tau(s), \varrho(s), s(x)$ by the corresponding functions.

In this article we analyse an important class of exactly solvable Schrödinger equations, but the class of known solvable problems is larger [3, 1,12 , 13, 14. Generally, the methods used to enlarge the class of exactly solvable potentials are based on the idea of finding pairs of (essentially) isospectral operators, and the construction of new exactly solvable Hamiltonians starts from a known exactly solvable Hamiltonian. In most of the cases the starting Hamiltonian belongs to the class considered in the present article. 
We have already seen that the superpotential $W_{m}$ which allows to construct the supersymmetric partner $V_{m+1}$ of $V_{m}$ satisfies the Riccati equation

$$
V_{m}(x)-\lambda_{m}=W_{m}^{2}(x)-\dot{W}_{m}(x) .
$$

New supersymmetric partners of $V_{m}$ can be obtained by finding new solutions $W$ of this equation [12, 13] or by solving the more general Riccati equation [3, 17

$$
V_{m}(x)-\varepsilon=W^{2}(x)-\dot{W}(x) .
$$

The usual algebraic approach can also be extended to these new exactly solvable potentials by using some non-linear generalizations of Lie algebras 4 . Certain algebraic properties become more transparent if we use the method proposed recently by Gurappa et. al. [9] which allows to connect the space of the solutions of a linear differential equation to the space of monomials, but the advantages obtained in the case of our Hamiltonians are not very significant.

\section{References}

[1] Antoine J-P, Gazeau J-P, Monceau P, Klauder J R and Penson K A 2001 Temporally stable coherent states for infinite well and Pöschl-Teller potentials J. Math. Phys. 42 2349-87

[2] Balantekin A B 1998 Algebraic approach to shape invariance Phys. Rev. A 57 4188-91

[3] Cannata F, Junker G and Trost J 1998 Solvable potentials, non-linear algebras, and associated coherent states Los Alamos preprint quant-ph/9806080

[4] Chaturvedi S, Dutt R, Gangopadhyaya, Panigrahi P, Rasinariu C and Sukhatme U 1998 Algebraic shape invariant models Phys. Lett. A 248 109-13

[5] Cooper F, Khare A and Sukhatme U 1955 Supersymmetry and quantum mechanics Phys. Rep. $251267-385$

[6] El Kinani A H and Daoud M 2002 Generalized coherent and intelligent states for exact solvable quantum systems J. Math. Phys. 43 714-33

[7] Fakhri H and Seyed Yagoobi S K A 2001 A master function approach for describing shape invariance parameters J. Phys. A: Math. Gen. 34 9861-69

[8] Fukui T and Aizawa N 1993 Shape-invariant potentials and an associated coherent state Phys. Lett. A $180308-13$

[9] Gurappa N, Panigrahi P K and Shreecharan T 2002 Linear differential equations and Orthogonal Polynomials: A novel approach Los Alamos preprint quant-ph/0203015

[10] Infeld L and Hull T E 1951 The factorization method Rev. Mod. Phys. 23 21-68

[11] Jafarizadeh M A and Fakhri H 1998 Parasupersymmetry and shape invariance in differential equations of mathematical physics and quantum mechanics Ann. Phys., NY 262 260-76

[12] Junnker G and Roy P 1997 Conditionally exactly solvable problems and non-linear algebras Phys. Lett. A 232 155-61

[13] Junnker G and Roy P 1998 Conditionally exactly solvable potentials: A supersymmetric construction method Los Alamos preprint quant-ph/9803024

[14] Lipan O and Rasinariu C 2002 Baxter T-Q equation for shape invariant potentials. The finite-gap potentials case J. Math. Phys. 43 847-65

[15] Lorente M 2001 Raising and lowering operators, factorization and differential/difference operators of hypergeometric type J. Phys. A: Math. Gen. 34 569-88

[16] Lorente M 2001 Continuous vs discrete models for the quantum harmonic oscillator and the hydrogen atom Phys. Lett. A 285 119-26

[17] Mielnik B, Nieto L M and Rosas-Ortiz O 2000 The finite difference algorithm for higher order supersymmetry Phys. Lett. A 269 70-8

[18] Nikiforov A F, Suslov S K and Uvarov V B 1991 Classical Orthogonal Polynomials of a Discrete Variable (Berlin: Springer) pp 2-17

[19] Richtmyer R D 1978 Principles of Advanced Mathematical Physics (New-York: Springer) pp $68-221$

[20] Whittaker E T and Watson G N 1950 A Course of Modern Analysis (Cambridge: Cambridge University Press) p 325 